\title{
New Hybrid Cutting Plane Method For Solving Integer Linear Programming
} Problems

Abbas Y. Al-Bayati

profabbasal-bayati@yahoo.com

Nawar N. Abdullah

\section{College of Computer Sciences and Mathematics \\ University of Mosul/Iraq}

Received: $22 / 3 / 2005$

Accepted: 29/6/2005

\section{ABSTRACT}

This work deals with a new method for solving Integer Linear Programming Problems depending on a previous methods for solving these problems such that Branch and Bound method and Cutting Planes method where this new method is a combination between them and we called it Cut and Branch method, The reasons which led to this combination between Cutting Planes method and Branch and Bound method are to defeat from the drawbacks of both methods and especially the big number of iterations and the long time for the solving and getting of a results between the results of these methods where the Cut and Branch method took the good properties from the both methods.

And this work deals with solving a one problem of Integer Linear Programming Problems by Branch and Bound method and Cutting Planes method and the new method, and we made a programs on the computer for solving ten problems of Integer Linear Programming Problems by these methods then we got a good results and by that, the new method (Cut and Branch) became a good method for solving Integer Linear Programming Problems.

The combination method which we doing in this research opened a big and wide field in solving Integer Linear Programming Problems and finding the best solutions for them where we did the combination method again between the new method (Cut and Branch) and the Cutting Planes method then we got a new method with a very good results and solutions.

Keywords: Integer Linear Programming, Cutting Plane Method, Cut and Branch method.

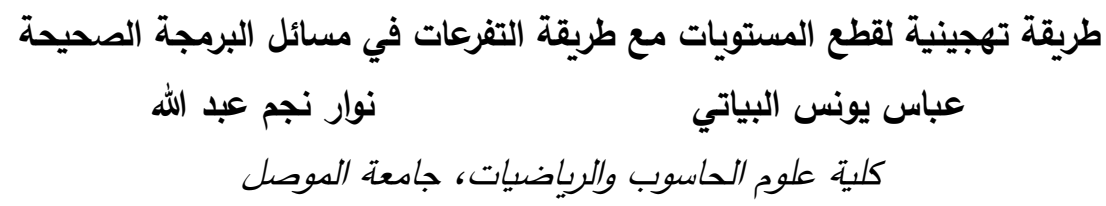

تاريخ قبول البحث: 29 / 6 | 2005

تاريخ استلام البحث: 22 / 3 / 2005

(الملخص

يتتاول هذا البحث طريقة جديدة في حل مسائل البرمجة الخطية الصحيحة بالاعتماد على طرق سابقة في

حل هذه المسائل منها طريقة التقرعات والعقد وطريقة قطع المستويات المعروفتين حيث تم في هذه الطريقة الجديدة ربط ما بين تلك الطريقتين وسميناها بطريقة القطع والتفرع.

الأسباب التي أدت إلى الربط مـا بين طريقة قطع المستويات وطريقة التقرعات والعقد هي للتغلب على

المساوئ من كلا الطريقتين وخاصة عدد التكرارات الكبير والوقت المستغرق الكثير في الحل والحصول على نتائج

تقع ما بين نتائج كل من تلك الطريقتين حيث أخذت طريقة القطع والتفرع الصفات الجيدة وجزء بسيط من الصفات

السيئة من كلا الطريقتين.

الكلمات المفتاحية: البرمجة الصحيحة، طريقة قطع المستويات، طريقة القطع والتقرع. 


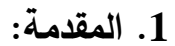

مسألة البرمجة الصحيحة Integer Programming Problem هي إحدى أفرع البرمجة الرياضية

Mathematical Programming(MP)

لدالة تتكون من عدة متغيرات وتحقق عدداً من القيود (معادلات أو متراجحات عادة) والتي يمكن تمثيلها بالثكل: MP: $\max (\min ) f(x) ; x \in S \subseteq R^{n}$

حيث أن المجموعـة S تسمى بمجموعـة القيود (Constraints Set و f (Feasible Solution) وكل اللبرمجة الرياضية. إذا (Objective Function) كان هناك لكل $x \in S$ فأن $x^{*}$ تسمى بالحل الأمثل (Optimal Solution) لكسألة البرمجة الرياضية. مسألة البرمجة الخطية Linear Programming Problem (LPP) هي حالة خاصـة من البرمجة الرياضية مع الدالة (x) f تكون دالة خطية و S تكون مجموعة من المتغيرات x تحقق منظومة من المعادلات الخطيـة وإن $Z^{n} \subseteq R^{n} \subseteq S$ على أن تكون ذات قيم صحيحة). [4] مسألة البرمجة الصحيحة Integer Programming Problem تكتب للاختصـار (IPP) بدأت في 1958 من قبل العالم (Ralph E. Gomory) وهي مسألة أمثلية لتكبير أو لتصغير قيمة دالة مع متغيرات القرار [2] تحقق مجموعة من المتراجحات. (Decision variables) يوجد هنالك نوعان أساسيان من مسألة البرمجة الصحيحة وهما:

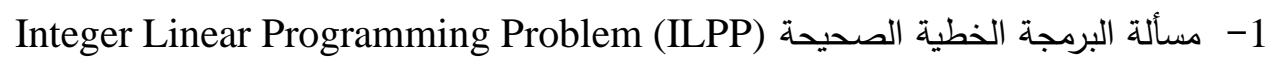

2- مسألة البرمجة اللاخطية الصحيحة Integer Nonlinear Programming Problem (INLPP) والفرق في ما بينهما هو خطية و لاخطية دالة الهدف.

مسألة البرمجة الخطية الصحيحة Integer Linear Programming Problem (ILPP) مسألة البرمجة الخطية الصحيحة هي مسألة البرمجة الخطية بحيث تكون جميع أو بعض متغيرات القرار مقيدة لتكون ذات قيمة صحيحة، و هناك ثلاثة أنواع من مسألة البرمجة الخطية الصحيحة: 1- مسألة البرمجة الخطية الصحيحة المطلقة Pure Integer Programming Problem (PIPP)

$$
\text { وهي المسألة التي تكون فيها جميع متغيرات القرار ذات قيمة صحيحة. }
$$

2- مسألة البرمجة الخطية الصحيحة المختلطة) Mixed Integer Programming Problem (MIPP) وهي المسألة التي تكون فيها بعض متغيرات القرار ذات قيمة صحيحة و بعضها الآخر ذات قيمة غير

$$
\text { صحيحة. }
$$

3- مسألة البرمجة الخطية الصحيحة الثنائية Binary Integer Programming Problem (BIPP)

وهي المسألة التي تكون فيها جميع متغيرات القرار ذات قيمة صفر أو واحد. [7] 2. طريقة التفرعات والعقد: 
طريقة التفرعات والعقد Branch and Bound method (B.B.) هي تقنية عددية منتظمة لحل مسائل

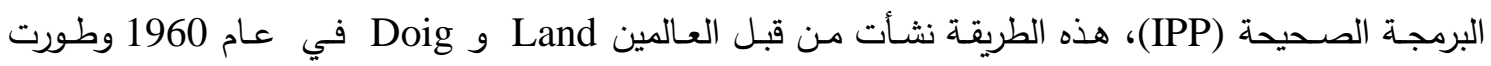
بوساطة العالم Dakin في العام 1965، خوارزمية طريقة التفرعات والعقد على مدى تحسيناتها وتوسعاتها

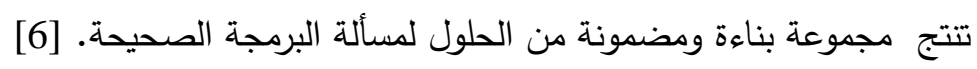
تسمية طريقة التفرعات و العقد جاءت من (التثرع) الذي يحدث على العقد المختارة لنمو أكثر وتكوين العقد التداء الجديدة (الأبناء) من هذه العقدة و(التقيد) الذي يحصل عند حساب القيد على أفضل قيمة تم الحصول عليها من

نمو العقد. [1] 2.1 خوارزمية طريقة التفرعات والعقد : لتكن مسألة البرمجة الصحيحة هي كالأتي:

$$
\left.\begin{array}{rl}
\text { Maximize } z & =c^{T} x \\
\text { Subject to } A x & =b \\
x & \geq 0 \\
x_{j}, \quad j \in I, \text { int egral }
\end{array}\right\}
$$

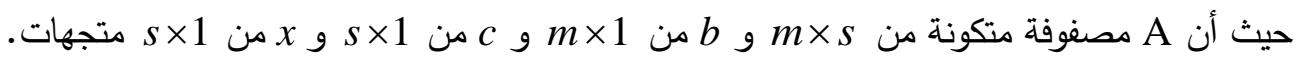

الخوارزمية:

الخطوة 1: (الحل الابتدائي) أبدأ بحل المسألة المعطاة في (1) على أنها مسألة البرمجة الخطية بطريقة سمبلكس (Simplex Method) .

الخطوة 2: (تفرع المتغيرات المختارة) اختر من المتغيرات تلك العقدة، متغير واحد يستخدم لتكوين قيود التفرع، قاعدة بسيطة في ذلك الاختيار هي اختيار المتغير التي تكون قيمته لها أكبر جزء كسري. المتغيرات المختارة x $x_{j}$ يجب أن تكون متغيرات أساسية وإلا قيمتها ستكون صغراً. افرض إن المتغير الأساسي المختار هو المتغير i من الجدول الأخير من حل مسألة البرمجة الخطية وتكون قيمته هي $x_{B i}$ ، حيث نستطيع كتابتها على الثكل التالي x x x

$x_{j} \leq\left[x_{B i}\right]$

$x_{j} \geq\left[x_{B i}\right]+1$

الخطوة 3: (صياغة العقد الجديدة) اعمل على تكوين مسألتين جديدتين من مسائل البرمجة الصحيحة متمثلتين بالعقد تحت الوصف في الخطوة 2. المسألة الأولى متكونـة من إضـافة القيد (2) والمسألة الأخرى متكونـة من إضـافة القيد (3)، حل كل من هاتين المسألتين على أنهما مسائل البرمجة الخطية مستخدما طريقة سمبلكس. 
الخطوة 4: (اختبار العقدة النهائية) كل من العقد المتكونة في الخطوة 3 ربما تكون عقدة نهائية لواحد من السببين الآتيين:

1- المسألة عند تلك العقدة ليس لها حل ممكن واذهب إلى الخطوة 5. 2- قيم المتغيرات الـ توصلت إليها، إذا كانت قيمة دالة الهدف عند العقدة الجديدة أفضل فغير قيمة العقدة القديمة بهذه العقدة واذهب إلى الخطوة 5. الخطوة 5: (اختيار العقدة)

1- إذا كانت بالضبط عقدة واحدة في الخطوة 4 منتهية، استخدم العقدة غير المنتهية واذهب إلى الخطوة 2.

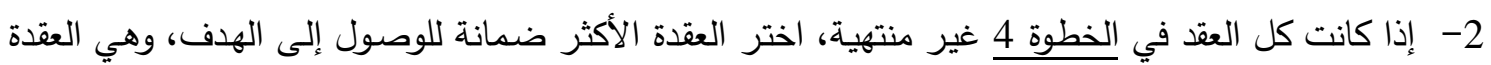
التي تكون عندها دالة الهدف اكبر ما يمكن، اذهب إلى الخطوة 2.

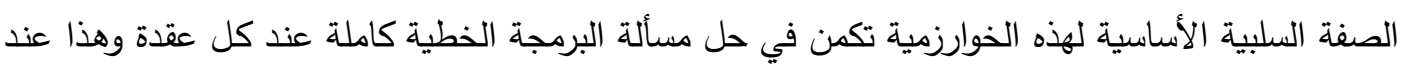
المسائل الكبيرة سيأخذ وقت كبير وعدد تكرارات اكبر [5]. 2.2 خواص طريقة التفرعات والعقد: إيجابيات طريقة التفرعات والعقد: 1) الوقت المستغرق في الحل Central Processing Unit (CPU) قليل. 2) يتم الحصول على الحل بوساطة التجزئة وذلك بتجزئة (تفرع) المتغيرات ذات القيم الحقيقية من الجدول الأخير لحل مسألة البرمجة الخطية ونستمر بالتجزئة إلى حين الحصول على الحل الأمثل بالاعتماد على طريقة سمبلكس. 3) طريقة التفرعات والعقد طريقة مضمونة الوصول إلى الهدف. سلبيات طريقة التفرعات و العقد:

1) عدد التكرارات Number of Iterations (NOI) في الحل كبير وخاصة عند البدء بتفرع قيمة غير كفوءة. 2) يجب الاستمرار بالتفرع حتى ولو حصلنا على الحل إلى حين الحصول على عقد مكرة و خارجة عن المنطقة. 3) عدد العقد المولدة في طريقة التفرعات والعقد ممكن أن يكون عددا كبيرا. 3. طريقة قطع المستويات:

طريقة قطع المستويات Cutting Planes method (C.P.) هي طريقة فريدة من نوعها، وهي الطريقة

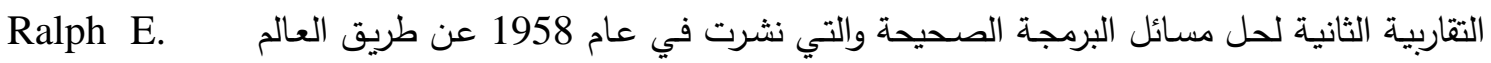

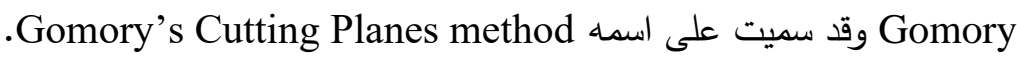
الفكرة الأساسية لطريقة قطع المستويات هي إضـافة قيد (قاطع) للمسألة مرة تلو الأخرى إلى أن تتكون مسألة البرمجة الخطية مع الحل الأمثل مع القيم الصحيحة. لهذا القيد خاصتان أساسيتان: الأولى، الحل الأمثل غير الصحيح لهئل الصألة البرمجة الخطية لن يحقق هذا

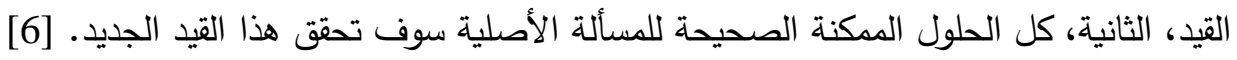
3.1 خوارزمية طريقة قطع المستويات: 
Maximize $z=c^{T} x$

لتكن مسألة البرمجة الصحيحة هي كالأتي:

Subject to $A x=b$

$$
x \geq 0
$$

$x_{j}, \quad j \in I$, int egral

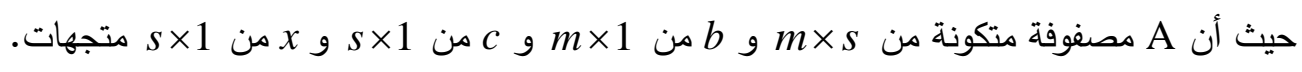

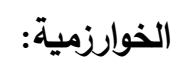

الخطوة 1: (الحل الابتدائي) ابدأ بحل المسألة المعطاة في (4) على أنها مسألة البرمجة الخطية بطريقة سمبلكس (متجاهلا بذلك الثروط (القيود) الصحيحة على المتغيرات. إذا كانت جميع المتغيرات (Simplex Method)

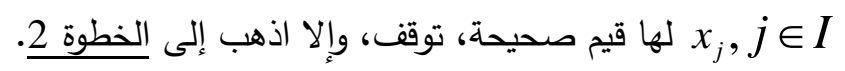

الخطوة 2: (اختيار القيد) اختر السطر من الجدول الأخير من حل مسألة البرمجة الخطية الذي يكون فيه المتغير الأساسي $x_{B i}$ ربما يساعد على تقليل عدد التكرارات والوقت المستغرق للتقارب) ومنه قم بتوليد قيد قطع المستويات. الخطوة 3: (توليد قيد قطع المستويات) افرض أن السطر المختار هو السطر $i$ ومعادلته هي: $x_{B i}+\sum_{j} a_{i j} x_{j}=b_{i}, j \in I \quad$ فان

$x_{B i}+\sum_{j}\left(\left[a_{i j}\right]+f_{i j}\right) x_{j}=\left[b_{i}\right]+f_{i}$

$x_{B i}+\sum_{j}\left[a_{i j}\right] x_{j}-\left[b_{i}\right]=f_{i}-\sum_{j} f_{i j} x_{j} \leq 0$

$f_{i}-\sum_{j} f_{i j} x_{j}+\delta=0$

القيد الجديد

$$
\begin{aligned}
& \text { حيث } 0 \leq f_{i j}<1 \quad a_{i j} \\
& b_{i} \text { ل } 0 \leq f_{i}<1
\end{aligned}
$$

ס متغير منحل جديد ممكن وصحيح.

الخطوة 4: (إضافة القيد) قم بإضافة القيد (5) للجدول الأخير من حل طريقة سمبلكس وقم بالحل على أنها مسألة

البرمجة الخطية، إذا كانت جميع المتغيرات

3.2 خواص طريقة قطع المستويات:

إيجابيات طريقة قطع المستويات:

1

2) يتم الحصول على الحل بوساطة القواطع التي يمكن استخراجها من الجدول الأخير لحل مسألة البرمجة الخطية حيث يتم قطع منطقة الحل إلى حين الحصول على الحل الأمثل بالاعتماد على طريقة سمبلكس. سلبيات طريقة قطع المستويات: 


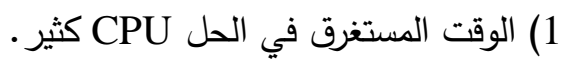

2) في بعض الأحيان لا نحصل على الحل وخاصـة حين البدء بالحل بأحد القواطع غير الكفوءة وبذلك تكون

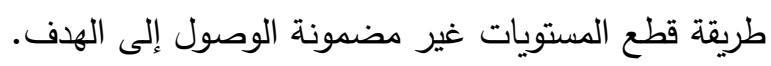
3) عدد القيود المولدة في طريقة قطع المستويات مدكن أن يكون كبيرا.

\title{
4. طريقة القطع والتفرع (الجديدة):
}

طريقة القطع و التفرع (Cut and Branch method (C.B.) هي تقنية ناجحة جدا في حل مجموعة واسعة من مسائل البرمجة الصحيحة وهي توفر ضمانية الوصول إلى الحل الأمثل. وسوف نقوم بتوضيح كيفية أداء هذه التقنية.

مساحة خوارزمية طريقة القطع والتقرع مستمرة التطور وهي واعدة بان تكون ذات أهمية أكبر مع استغلال

$$
\text { سرعة الحاسوب. }
$$

عدد كبير من مسائل البرمجة الخطية الصحيحة ممكن حلها بوساطة طريقة القطع والتفرع والتي تكون

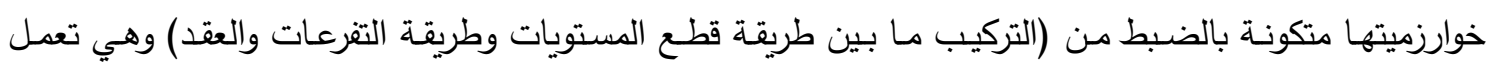
كسابقتها في حل سلسلة (متتابعة) من مسائل البرمجة الخطية للحصول على حل مسألة البرمجة الصحيحة.

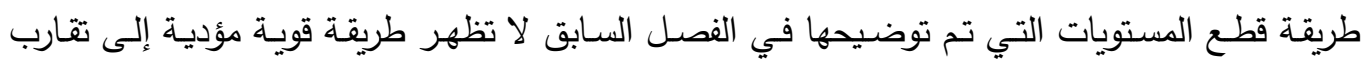

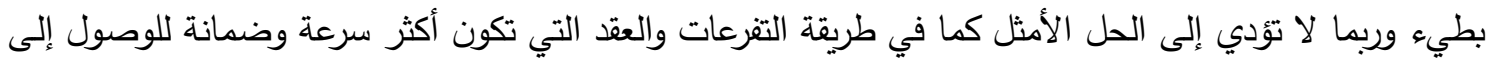
الحل الأمثل لذلك حاولنا جعل طريقة قطع المستويات أفضل من السـابق بوساطة طريقة التثرعات والعقد والتي أسميناها بطريقة القطع والتقرع.

\section{1 خوارزمية طريقة القطع والتفرع:} لتكن مسألة البرمجة الصحيحة هي كالأتي:

\author{
Maximize $z=c^{T} x$ \\ Subject to $A x=b$ \\ $x \geq 0$ \\ $x_{j}, \quad j \in I$, int egral \\ حيث أن A مصفوفة متكونة من ×s و
}

الخطـوة 1: (الحـل الابتـدائي) ابـدأ بحل المسـألة المعطـاة في (6) على أنها مســألة البرمجـة الخطيـة بطريقـة سمبلكس (Simplex Method) متجاهلا بذلك الشروط (القيود) الصـحيحة على المتغيرات. إذا كانت جميع المتغيرات الخطوة 2: (اختيار القيد) اختر السطر من الجدول الأخير من حل مسألة البرمجة الخطية الذي يكون فيه المتغير الأساسي $x_{B i}$ ربما يساعد على تقليل عدد التكرارات والوقت المستغرق للتقارب) ومنه قم بتوليد قيد قطع المستويات. الخطوة 3: (توليد قيد قطع المستويات) افرض أن السطر المختار هو السطر i ومعادلته هي: 


$$
\begin{aligned}
& x_{B i}+\sum_{j} a_{i j} x_{j}=b_{i}, j \in I \\
& x_{B i}+\sum_{j}\left(\left[a_{i j}\right]+f_{i j}\right) x_{j}=\left[b_{i}\right]+f_{i} \\
& x_{B i}+\sum_{j}\left[a_{i j}\right] x_{j}-\left[b_{i}\right]=f_{i}-\sum_{j} f_{i j} x_{j} \leq 0 \\
& f_{i}-\sum_{j} f_{i j} x_{j}+\delta=0
\end{aligned}
$$

القيد الجديد

$$
\begin{aligned}
& \text { حيث } 0 \leq f_{i j}<1 \quad a_{i j} \\
& \left.b_{i}\right\rfloor \text { 」 } 0 \leq f_{i}<1 \\
& \text { Sتغير منحل جديد ممكن وصحيح. }
\end{aligned}
$$

الخطوة 4: ( إضـافة القيد) قم بإضـافة القيد (7) إلى الجدول الأخير من حن حل طريقة سمبلكس لمسألة البرمجـة الخطية وحلها على أنها مسألة البرمجة الخطية، إذا كانت: 1- المتغيرات

2- متغيرات 3- متغير واحد من المتغيرات

$$
\text { المتغير غير الصحيح أي اذهب إلى الخطوة 5. }
$$

الخطوة 5: (اختيار المتغير المتفرع) اختر المتغير ذا القيمة غير الصحيحة من لتكوين قيود التفرع والذي له أكبر جزء كسري.

المتغير $x_{j}$

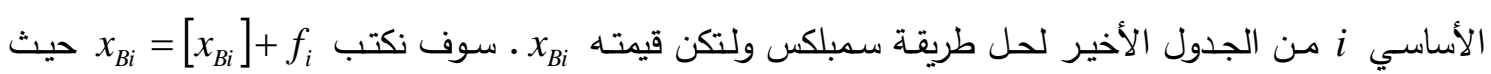
> 0 . بما أن المتغير $x_{i}<1$

$x_{j} \leq\left[x_{B i}\right]$

$x_{j} \geq\left[x_{B i}\right]+1$

الخطوة 6: (صيغة العقدة الجديدة) كوّن مسألتين جديدتين من مسائل البرمجة الصحيحة بالقيود المتمثلة في الخطوة 5. مسألة واحدة متكونـة من إضـافة القيد (8) والمسألة الأخرى متكونـة من إضـافة القيد (9) وحل كل كل من هاتين المسألتين على أنهما مسائل البرمجة الخطية باستخدام طريقة سمبلكس.

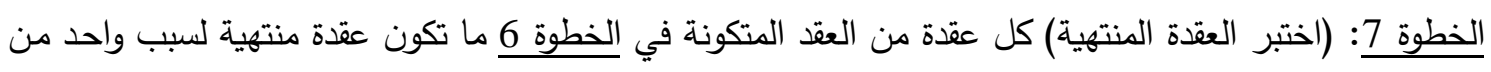
الأسباب الآتية، أولا، المسألة المتمثلة بهذه العقدة ليس لها حل ممكن، ثانيا، قيمة المتغيرات صحيحة وهذا هو الحل الأمثل للمسألة (6). أما إذا كانت تلك العقدة غير منتهية فأرجع إلى الخطوة 5. 4.2 خوارزمية ثانية إلى طريقة القطع والتفرع (طريقة القطع والتفرع والقطع): لتكن مسألة البرمجة الصحيحة هي كالأتي: 
Maximize $z=c^{T} x$

Subject to $A x=b$

$x \geq 0$

$x_{j}, \quad j \in I$, int egral

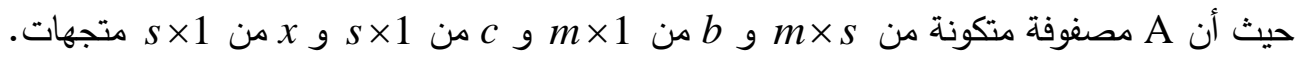

الخوارزمية:

الخطوة 1: (الحل الابتدائي) ابدأ بحل المسألة المعطاة في (10) على أنها مسألة البرمجة الخطية بطريقة سمبلكس (Simplex method) . الخطوة 2: (اختيار القيد) اختر السطر من الجدول الأخير من حل مسألة البرمجة الخطية الذي يكون فيه المتغير

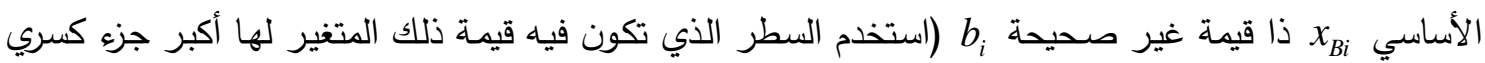
ربما يساعد على تقليل عدد التكرارات والوقت المستغرق للتقارب) ومنه قم بتوليد قيد قطع المستويات. الخطوة 3: ( توليد قيد قطع المستويات) افرض أن السطر المختار هو السطر i $i$ ومعادلته هي: $x_{B i}+\sum_{j} a_{i j} x_{j}=b_{i}, j \in I \quad$ فان

$x_{B i}+\sum_{j}\left(\left[a_{i j}\right]+f_{i j}\right) x_{j}=\left[b_{i}\right]+f_{i}$

$x_{B i}+\sum_{j}\left[a_{i j}\right] x_{j}-\left[b_{i}\right]=f_{i}-\sum_{j} f_{i j} x_{j} \leq 0$

$f_{i}-\sum_{j} f_{i j} x_{j}+\delta=0$

القيد الجديد

$$
\begin{aligned}
& \text { حيث } 0 \leq f_{i j}<1 \quad a_{i j} \text { لـ } \\
& b_{i} \text { لـ } 0 \leq f_{i}<1
\end{aligned}
$$$$
\text { } \delta \text { متغير منحل جديد ممكن و صحيح. }
$$

الخطوة 4: (إضـافة القيد) قم بإضـافة القيد (11) إلى الجدول الأخير من حل طريقة سمبلكس لمسألة البرمجـة الخطية وحلها على أنها مسألة البرمجة الخطية، إذا كانت: 1- كل المتغيرات 2- كل المتغيرات 3- متغير واحد من المتغيرات رئI, المتغير غير الصحيح أي اذهب إلى الخطوة 5. الخطوة 5: (اختيار المتغير المتفرع) اختر المتغير ذا القيمـة غير الصـيحة من الكن لتكوين قيود التقرع والذي لله أكبر جزء كسري. 
المتغير x الدختار يجب أن يكون متغيرا أساسيا وإلا ستكون قيمته صفرا. افرض أن المتغير هو المتغير

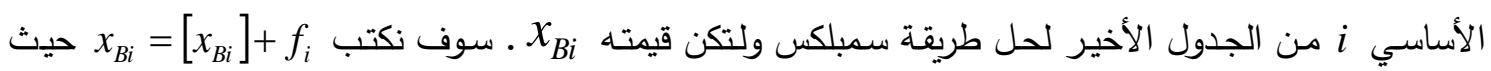

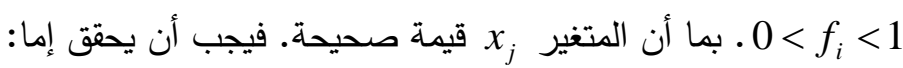

$x_{j} \leq\left[x_{B i}\right]$

$x_{j} \geq\left[x_{B i}\right]+1$

الخطوة 6: (صيغة العقدة الجديدة) كوّن مسألثين جديدتين من مسائل البرمجة الصحيحة بالقيود الكتمثلة في الخطوة

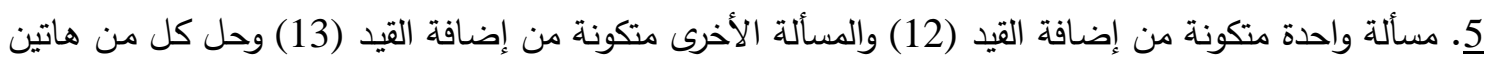

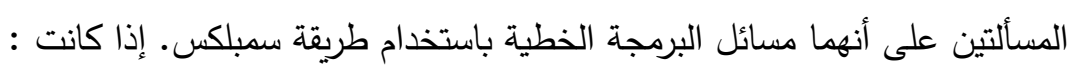
1- كل المتغيرات الهن

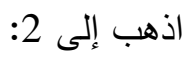

2- الحل غير ممكن أي خارج منطقة الحل عندها توقف وخذ عقدة أخرى وإلا اذهب إلى 3:

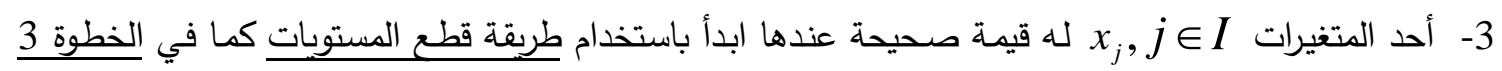

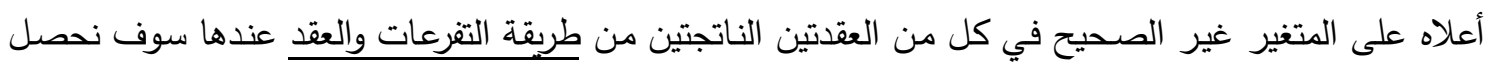
على الحلّين الآتيين من كل عقدة:

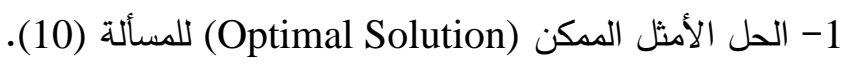
2- الحل غير ممكن (Infeasible Solution) عندها توقف.

4.3 خواص طريقة القطع والتفرع:

1) عدد التكرارات NOI قليل (ما بين عدد تكرارات كل من طريقة قطع المستويات والتثرعات والعقد).

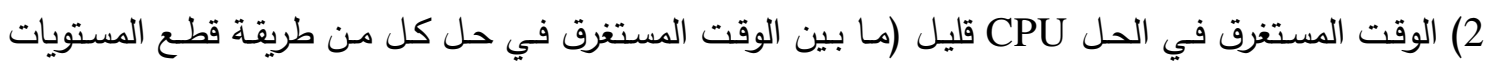
والتثرعات والعقد). 3) يتم الحصول على الحل أولا بوساطة القواطع التي يمكن استخراجها من الجدول الأخير لحل مسألة البرمجة

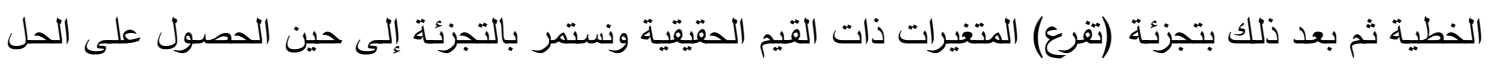
الأمثل بالاعتماد على طريقة سمبلكس. 4) أصبحت طريقة مضمونة الوصول إلى الهدف لأنها تنتهي بالحل بطريقة التنرعات والعقد.

5. - 5. النتائج العددية والعمل المستقبلي: 5.1

في هذه البحث تم اختيار عشرة مسائل [3] من البرمجة الخطية الصحيحة المطلقة وحلها بالحاسوب لأجل ملاحظة مدى دقة النتائج ونجاح طريقة الحل الجديدة وهذه المسائل هي كالآتي: 
الجدول (1).

\begin{tabular}{|c|c|c|c|c|c|c|c|}
\hline \multirow[b]{2}{*}{$\mathbf{E}$} & \multirow{2}{*}{$\begin{array}{lllllll}\mathbf{x} & \mathbf{a} & \mathbf{m} & \mathbf{p} & \mathbf{l} & \mathbf{e} & \mathbf{s}\end{array}$} & \multicolumn{2}{|c|}{ dBranch and Boun } & \multicolumn{2}{|c|}{ sCutting Plane } & \multicolumn{2}{|c|}{$\begin{array}{ll}\mathbf{W} & \mathbf{E} \\
\end{array}$} \\
\hline & & IN O & $\begin{array}{r}\text { UCP } \\
)(\sec \end{array}$ & IN O & )CPU (sec & IN O & CPU (sec) \\
\hline$Q 1$ & $\begin{array}{l}\text { Max } Z=5 x_{1}+2 x_{2} \\
\text { Subject to } \\
5 x_{1}+4 x_{2} \leq 21 \\
x_{1}, x_{2} \geq 0, \text { integers }\end{array}$ & 4 & 40.2 & 3 & 60.2 & 3 & 60.4 \\
\hline$Q 2$ & $\begin{array}{l}\text { Max } Z=10 x_{1}+x_{2} \\
\text { Subject to } \\
2 x_{1}+5 x_{2} \leq 11 \\
x_{1}, x_{2} \geq 0, \text { integers }\end{array}$ & 4 & 20.2 & 2 & 80.3 & 3 & 90.2 \\
\hline$Q 3$ & $\begin{array}{l}\operatorname{Max} Z=4 x_{1}+3 x_{2} \\
S u b j e c t \quad t \quad \\
3 x_{1}+2 x_{2} \leq 18 \\
x_{1}, x_{2} \geq 0 \text {, integers }\end{array}$ & 2 & 10.1 & 1 & 2.30 & 1 & 00.2 \\
\hline$Q 4$ & $\begin{array}{l}\text { Max } Z=120 x_{1}+80 x_{2} \\
\text { Subject to } \\
2 x_{1}+x_{2} \leq 6 \\
7 x_{1}+8 x_{2} \leq 28 \\
x_{1}, x_{2} \geq 0, \text { integers }\end{array}$ & 7 & 30.3 & 4 & 00.9 & 5 & 00.7 \\
\hline$Q 5$ & $\begin{array}{l}\text { Max } Z=5 x_{1}+8 x_{2} \\
\text { Subject to } \\
x_{1}+x_{2} \leq 6 \\
5 x_{1}+9 x_{2} \leq 45 \\
x_{1}, x_{2} \geq 0, \text { integers }\end{array}$ & 8 & 70.3 & 3 & 70.8 & 3 & 50.6 \\
\hline$Q 6$ & $\begin{array}{l}\text { Max } Z=3 x_{1}+x_{2} \\
\text { Subject to } \\
x_{1}+2 x_{2} \leq 8 \\
3 x_{1}-4 x_{2} \leq 12 \\
x_{1}, x_{2} \geq 0, \text { integers }\end{array}$ & 9 & 90.4 & 4 & 31.0 & 5 & 40.7 \\
\hline$Q 7$ & $\begin{array}{l}\text { Max } Z=4 x_{1}+3 x_{2} \\
\text { Subject to } \\
2 x_{1}+x_{2} \leq 11 \\
-x_{1}+2 x_{2} \leq 6 \\
x_{1}, x_{2} \geq 0, \text { integers }\end{array}$ & 7 & 10.3 & 4 & 80.9 & 6 & 70.7 \\
\hline$Q 8$ & $\begin{array}{l}\text { Max } Z=6 x_{1}+7 x_{2} \\
\text { Subject to } \\
x_{1}+2 x_{2} \leq 8 \\
x_{1}-x_{2} \leq 4 \\
2 x_{1}+2 x_{2} \leq 5 \\
x_{1}, x_{2} \geq 0 \text {, integers }\end{array}$ & 9 & 20.7 & 2 & 71.0 & 3 & 40.8 \\
\hline
\end{tabular}




\begin{tabular}{|c|c|c|c|c|c|c|c|}
\hline$Q 9$ & $\begin{array}{l}\operatorname{Max} Z=x_{1}+9 x_{2}+x_{3} \\
\text { Subject to } \\
x_{1}+2 x_{2}+3 x_{3} \leq 9 \\
3 x_{1}+2 x_{2}+2 x_{3} \leq 15 \\
x_{1}, x_{2}, x_{3} \geq 0, \text { integers }\end{array}$ & 5 & 80.3 & 2 & 50.8 & 3 & 60.5 \\
\hline \multirow[t]{2}{*}{$Q 10$} & $\begin{array}{l}\text { Max } Z=2 x_{1}+x_{2}-3 x_{3} \\
\text { Subject to } \\
x_{1}+2 x_{2}-x_{3} \leq 5 \\
-x_{1}+x_{2}+3 x_{3} \leq-2 \\
x_{1}, x_{2}, x_{3} \geq 0, \text { integers }\end{array}$ & 2 & 10.3 & 1 & 60.6 & 1 & 90.4 \\
\hline & Total & 75 & 83.4 & 62 & 87.6 & 33 & 05.7 \\
\hline
\end{tabular}

Performance percentage of improving the new algorithm compared with the two other standards

\begin{tabular}{|c|c|c|c|}
\hline Tools & NEW & $\begin{array}{c}\text { Branch and } \\
\text { Bound }\end{array}$ & Cutting Planes \\
\hline NOI & $\mathbf{1 0 0 \%}$ & $\mathbf{1 7 2 . 7 2 \%}$ & $\mathbf{7 8 . 7 9 \%}$ \\
\hline CPU & $\mathbf{1 0 0 \%}$ & $\mathbf{6 1 . 0 5 \%}$ & $\mathbf{1 3 4 . 7 3 \%}$ \\
\hline
\end{tabular}

من جدول النسبة المئوية السابق نلاحظ تحقق الصفات 1 و2 من صفات طريقة القطع والتقرع:

$$
\begin{aligned}
& \text { عدد تكرارات طريقة } \\
& \text { قطع المستويات }
\end{aligned}
$$

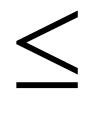

$$
\text { الوقت المستغرق لطريقة }
$$

CPU of Cutting

Planes
الوقت المستغرق

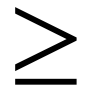

لطريقة القطع والتقرع

CPU of Cut and

Branch
الوقت المستغرق لطريقة

$\sum \begin{gathered}\text { CPU of Cutting and } \\ \text { التفرعات والعقد }\end{gathered}$

Bound

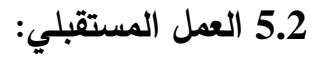

من هذا العمل نستنتج انه يمكن تركيب طريقتين للحصول على طريقة ثالثة ذات نتائج أفضل، فمن المكن ربط طريقة قطع المستويات وطريقة التقرعات والعقد أكثر من مرة كما لاحظنا في الفقرة 4.2 حيث تم الربط مرة أخرى بقطع المستويات أي حصلنا على (قطع وتفرع وقطع) فانتهى الناتج إلى حلين، حل خارج عن المنطقة (غير مكن) (Infeasible Solution) وحل أمثل للمسألة (Optimal Solution) فأصبح هذا التركيب الثلاثي الجديد تركيباً جيد جداً حيث أخذ الصفات الجيدة ما بين الطريقة الجديدة (القطع والتثرع) وطريقة قطع المستويات وهذا يفتح مستقبلا مجالا جديدا وواسعا في مسائل البرمجة الخطية الصحيحة (المطلقة والمختلطة) وإيجاد أفضل الحلول 


\section{المصادر}

[1] Chinneck, J. W. (2003): Practical optimization: A Gentle Introduction, Chapter12, pp.2-3, [http://www.sce.carleton.ca/faculty/ chinneck/po.html].

[2] Eudoxus System Ltd. (2003): Practical integer programming, Perth House Leighton Buzzard LU7 2RN UK, [http://www.eudoxus.com/lect5.pdf.].

[3] Gomory, R. E. (1973): An all-integer programming algorithm, Rand report, R.M. 25797, New York, Chapter 3, pp.46-66.

[4] Grafinkel, R. S. and Nemhauser, G. L. (2003): Integer programming, MATH3902 operation researches II, Chapter 1, pp.1-5.

[5] Taha, H. A. (1979): Operations research an introduction, Macmillan, New York, USA. Chapter 8, p.258.

[6] Thie, P. R. (1979): An introduction to linear programming and game theory, John Wiley Press, New York, USA, Chapter 6, p.187.

[7] Villalobos, J. R.; Hogg, G. L. and Griffin, P. M. (2002): Introduction to integer programming. Arizona state University and George institute of technology, pp.4-7. 